\title{
Utility of ICD Codes for Stress Cardiomyopathy in Hospital Administrative Databases: What Do They Signify?
}

\author{
Anusha G Bhat, MD',2, Kevin White, BS', Kyle Gobeil, DO, MSc1, Tara Lagu, MD, MPH',3, \\ Peter K Lindenauer, MD, MSc ${ }^{1,3}$, Quinn R Pack, MD, MSc ${ }^{1,3,4 *}$
}

\begin{abstract}
${ }^{1}$ Department of Medicine, University of Massachusetts Medical School, Baystate Medical Center, Springfield, Massachusetts; ${ }^{2}$ Division of Public Health Policy, School of Public Health and Health Sciences, University of Massachusetts, Amherst, Massachusetts; ${ }^{3}$ Division of Cardiovascular Medicine, University of Massachusetts Medical School, Baystate Medical Center, Springfield, Massachusetts; ${ }^{4}$ nstitute for Healthcare Delivery and Population Science at University of Massachusetts Medical School, Baystate, Springfield, Massachusetts.
\end{abstract}

Prior studies of stress cardiomyopathy (SCM) have used International Classification of Diseases (ICD) codes to identify patients in administrative databases without evaluating the validity of these codes. Between 2010 and 2016, we identified 592 patients discharged with a first known principal or secondary ICD code for SCM in our medical system. On chart review, 580 charts had a diagnosis of SCM (positive predictive value $98 \%$; $95 \%$ $\mathrm{Cl}$ : 96.4-98.8), although 38 (6.4\%) did not have active clinical manifestations of SCM during the hospitalization.
Moreover, only $66.8 \%$ underwent cardiac catheterization and $91.5 \%$ underwent echocardiography. These findings suggest that, although all but a few hospitalized patients with an ICD code for SCM had a diagnosis of SCM, some of these were chronic cases, and numerous patients with a new diagnosis of SCM did not undergo a complete diagnostic workup. Researchers should be mindful of these limitations in future studies involving administrative databases. Journal of Hospital Medicine 2020;15:160-163. (C) 2020 Society of Hospital Medicine tress cardiomyopathy (SCM), also known as takotsubo cardiomyopathy, is a nonischemic cardiomyopathy initially identified in Japan in 1990. In 2006, SCM gained an International Classification of Diseases code at the $9^{\text {th }}$ Clinical Modification (ICD-9 CM). Subsequently, several epidemiological studies have used ICD codes to evaluate trends in the diagnosis of $\mathrm{SCM}$; ${ }^{1-8}$ however, to our knowledge, no previous studies have validated ICD-9 or -10 codes using chart review. We aimed to determine the positive predictive value (PPV) and the limitations of these ICD codes among hospitalized patients.

\section{METHODS}

We performed a retrospective cohort study at a single tertiary care center, identifying all adults aged $\geq 18$ years from 2010 to 2016 who were hospitalized with a first known diagnosis of SCM in our Electronic Health Records (EHR) system (Cerner, Stoltenberg Consulting, Inc., Bethel Park, Pennsylvania), which includes both inpatient and outpatient records. We included patients hospitalized with a principal or secondary ICD-9 discharge diagnosis code of 429.83 (for those hospitalized before October 2015) or an ICD-10 discharge diagnosis code of I51.81

*Corresponding Author: Quinn R. Pack, MD, MSc; E-mail: Quinn.PackMD@ baystatehealth.org; Telephone: 413-794-5856

Published online first December 18, 2019

Received: May 17, 2019; Revised: October 16, 2019;

Accepted: October 20, 2019

() 2020 Society of Hospital Medicine DOI 10.12788/jhm.3344 (for those hospitalized from October 1, 2015 through December 2016). We excluded hospital readmissions and patients with recurrent SCM, but we could not administratively remove patients who carried a prior diagnosis of SCM made previously at other institutions. One investigator $(\mathrm{KW})$ then reviewed our EHR for a documentation of SCM anywhere in the chart by performing a systematic review of discharge, admission, consultation, daily progress notes, as well as biomarkers, electrocardiograms, echocardiograms, and coronary angiograms. If the first reviewer did not find documentation of SCM anywhere in the EHR, this finding was confirmed by a second chart review by a cardiologist (QP).

Principal and secondary discharge diagnoses were entered into our administrative database by hospital coders using standard coding practices. Because ICD codes also record comorbidities that were present prior to admission, we determined whether each patient had a new diagnosis of SCM during the hospitalization. If not, we considered their ICD code as a preexisting comorbidity and labeled these as chronic cases.

We recorded age, sex, race, ethnicity, and frequency of echocardiogram and cardiac catheterization among all patients. To determine the burden of other comorbidities, we used the Charlson Comorbidity Index and the Elixhauser Comorbidity Index, 9,10 but limited our reporting to comorbidities with $>5 \%$ prevalence.

Our primary aim was to measure the PPV of these ICD codes to determine a diagnosis of SCM. This was done by dividing the total number of cases with a clinical documentation of SCM by the total number of patients with an ICD diagnosis of SCM. As secondary aims, we noted the percentage of new and chronic 
SCM, the proportion of patients who underwent echocardiography and/or cardiac catheterization and recorded the annual number of total cases of confirmed SCM from 2010 to 2016. Trends were evaluated using the Cochran-Armitage test. To better understand the difference between patients given a principal and secondary code for SCM, we compared these two groups using summary statistics using $t$ tests and chi-squared tests as appropriate, noted the PPV, and determined the $95 \%$ confidence intervals of ICD codes in these subgroups. This study was approved by the institutional review board of Baystate Medical Center (\#1109756-4). Statistical analysis was done using JMP version12.0.1 (SAS Institute, Cary, North Carolina, 2015).

\section{RESULTS}

During 2010-2016, a total of 592 patients with a first known ICD code in our EHR for SCM were hospitalized, comprising 242 (41.0\%) with a principal diagnosis code. Upon chart review, we were unable to confirm a clinical diagnosis of SCM among $12(2.0 \%)$ patients. In addition, 38 (6.4\%) were chronic cases of SCM, without evidence of active disease at the time of hospitalization. In general, chronic cases typically carried an SCM diagnosis from a hospitalization at a non-Baystate hospital (outside our EHR), or from an outpatient setting. Occasionally, we also found cases where the diagnosis of SCM was mentioned but testing was not pursued, and the patient had no symptoms that were attributed to SCM. Overall use of echocardiogram and cardiac angiography was $91.5 \%$ and $66.8 \%$, respectively, and was lower in chronic than in new cases of SCM.

Compared with patients with a secondary diagnosis code, patients with a principal diagnosis of SCM underwent more cardiac angiography and echocardiography (Table 1). When comparing the difference between those with principal and secondary ICD codes, we found that 237 (98\%) vs 305 (87\%) were new cases of SCM, respectively, and all 12 patients without any clinical diagnosis of SCM had been given a secondary ICD code. Between 2010 and 2016, we noted a significant increase in the number of cases of SCM (Cochrane-Armitage, $P<.0001$ ).

The overall PPV $(95 \% \mathrm{Cl})$ of either principal or secondary ICD codes for any form or presentation of SCM was 98.0\% (96.498.8) with no difference in PPV between the coding systems (ICD-9, 66\% of cases, PPV 98\% [96.0-99.0] vs ICD-10, PPV 98\% [94.9-99.2; $P=.98])$. Because all patients without a diagnosis of SCM were given secondary ICD codes, this changed the PPV (95\% Cl) for principal and secondary SCM to 100\% (98.4-100.0) and $96.6 \%$ (94.1-98.0), respectively. When chronic cases were included as noncases, the PPV $(95 \% \mathrm{Cl})$ to detect a new case of SCM decreased to $97.9 \%$ (95.2-99.1) and 87.1\% (83.0-90.2) for principal and secondary SCM, respectively (Table 1 ).

\section{DISCUSSION}

In this study, we found a strong relationship between the receipt of an ICD code for SCM and the clinical documentation of a diagnosis of SCM, with an overall PPV of $98 \%$. The PPV was higher when the sample was limited to those assigned a principal ICD code for SCM, but it was lower when considering that some ICD codes represented chronic SCM from prior hospitalizations, de- spite our attempts to exclude these cases administratively prior to chart review. Furthermore, cardiac catheterization and echocardiography were used inconsistently and were less frequent among secondary compared with a principal diagnosis of SCM. Thus, although a principal ICD diagnosis code for SCM appears to accurately reflect a diagnosis of SCM, a secondary code for SCM appears less reliable. These findings suggest that future epidemiological studies can rely on principal diagnosis codes for use in research studies, but that they should use caution when including patients with secondary codes for SCM.

Our study makes an important contribution to the literature because it quantitates the reliability of ICD codes to identify patients with SCM. This finding is important because multiple studies have used this code to study trends in the incidence of this disease, ${ }^{1-8}$ and futures studies will almost certainly continue to do so. Our results also showed similar demographics and trends in the incidence of SCM compared with those of prior studies ${ }^{1-3,11}$ but additionally revealed that these codes also have some important limitations.

A key factor to remember is that neither a clinical diagnosis nor an ICD code at the time of hospital discharge is based upon formal diagnostic criteria for SCM. Importantly, all currently proposed diagnostic criteria require resolution of typical regional wall motion abnormalities before finalizing a research-grade diagnosis of SCM (Table 2). ${ }^{12,13}$ However, because the median time to recovery of ejection fraction in SCM is between three and four weeks after hospital discharge (with some recovery extending much longer), ${ }^{6}$ it is almost impossible to make a research-grade diagnosis of SCM after a threeto four-day hospitalization. Moreover, $33 \%$ of our patients did not undergo cardiac catheterization, $8.5 \%$ did not undergo echocardiography, and it is our experience that testing for pheochromocytoma and myocarditis is rarely done. Thus, we emphasize that ICD codes for SCM assigned at the time of hospital discharge represent a clinical diagnosis of SCM and not research-grade criteria for this disease. This is a significant limitation of prior epidemiologic studies that consider only the short time frame of hospitalization.

A limitation of our study is that we did not attempt to measure sensitivity, specificity, or the negative predictive value of these codes. This is because measurement of these diagnostic features would require sampling some of our hospital's 53,000 annual hospital admissions to find cases where SCM was present but not recognized. This did not seem practical, particularly because it might also require directly overreading imaging studies. Moreover, we believe that for the purposes of future epidemiology research, the PPV is the most important feature of these codes because a high PPV indicates that when a principal ICD code is present, it almost always represents a new case of SCM. Other limitations include this being a single-center study; the rates of echocardiograms, cardiac angiography, clinical diagnosis, and coding may differ at other institutions.

In conclusion, we found a high PPV of ICD codes for SCM, particularly among patients with a principal discharge diagnosis of SCM. However, we also found that approximately $8 \%$ of cases were either wrongly coded or were chronic cas- 
TABLE 1. Patient Demographics, Diagnostic Tests, Comorbidities, Trends, and Reliability by Diagnosis Codes

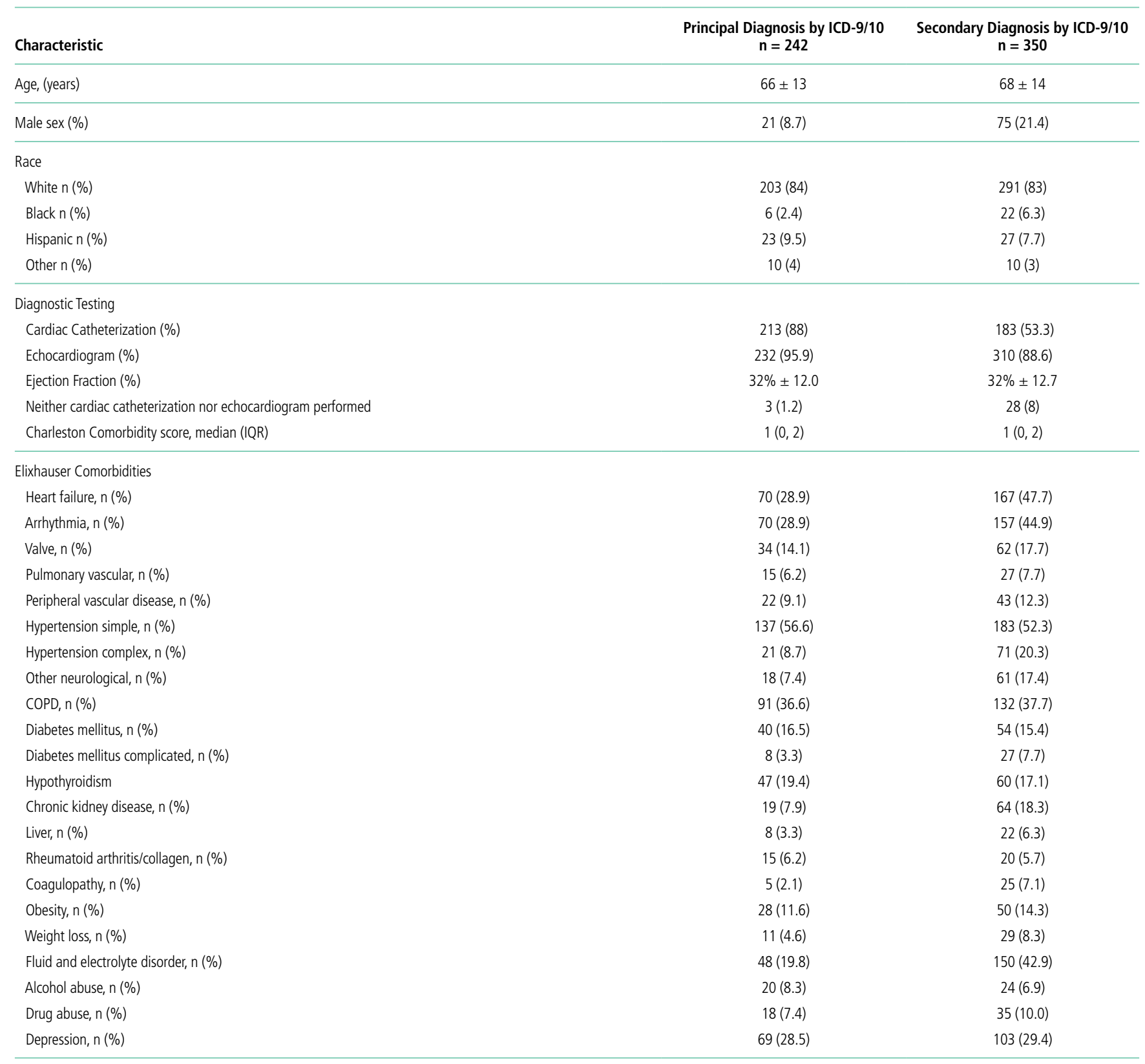

Yearly trends, $\mathrm{n}$ (cases per year)

\begin{tabular}{lcc}
2010 & 23 & 31 \\
2011 & 33 & 21 \\
2012 & 32 & 26 \\
2013 & 30 & 52 \\
2014 & 33 & 37 \\
2015 & 42 & 65 \\
2016 & 49 & 118 \\
\hline Reliability measures & & $0(0)$ \\
No documentation to support clinical diagnosis of stress cardiomyopathy, $n(\%)$ & $5(2.1)$ & $33(3.4)$ \\
Chronic cases without active manifestation of stress cardiomyopathy, $n(\%)$ & $237(97.9)$ & $305(87.0)$
\end{tabular}

Abbreviations: COPD, chronic obstructive pulmonary disease; ICD, International Classification of Diseases; IQR, interquartile range. 
TABLE 2. Comparison of Guideline Diagnostic Criteria for Stress Cardiomyopathy with Their Availability at the Time of Hospital Discharge

\begin{tabular}{|c|c|c|c|c|}
\hline Diagnostic Criteria & $\begin{array}{c}\text { Revised Mayo Clinic } \\
\text { Criteria }\end{array}$ & $\begin{array}{l}\text { International Takotsubo } \\
\text { Registry (InterTAK) }\end{array}$ & $\begin{array}{l}\text { ESC Heart Failure } \\
\text { Association }\end{array}$ & $\begin{array}{c}\text { Available at Time } \\
\text { of Hospital Discharge? }\end{array}$ \\
\hline Presence of typical regional wall motion abnormalities (RWMA) & + & + & + & Yes \\
\hline New EKG finding & + & + & + & Yes \\
\hline Changes in biomarkers & + & + & + & Yes \\
\hline Resolution of RWMA & + & + & + & Rarely \\
\hline Absence of obstructive CAD (cardiac catheterization) & + & - & + & Inconsistently available \\
\hline Absence of myocarditis & + & + & - & Rarely \\
\hline Absence of pheochromocytoma & + & - & - & Rarely \\
\hline
\end{tabular}

es. Moreover, because of the need to document resolution of wall motion abnormalities, essentially no patients met the research-grade diagnostic criteria at the time of hospital discharge. Although this increases our confidence in the results of past studies, it also provides some caution to researchers who may use these codes in the future.

\section{References}

1. Khera R, Light-McGroary K, Zahr F, Horwitz PA, Girotra S. Trends in hospitalization for takotsubo cardiomyopathy in the United States. Am Heart J. 2016;172:53-63. https://doi.org/10.1016/j.ahj.2015.10.022.

2. Murugiah $K$, Wang $Y$, Desai NR, et al. Trends in short- and long-term outcomes for takotsubo cardiomyopathy among medicare fee-for-service beneficiaries, 2007 to 2012. JACC Heart Fail. 2016;4(3):197-205. https://doi. org/10.1016/j.jchf.2015.09.013.

3. Brinjikji W, El-Sayed AM, Salka S. In-hospital mortality among patients with takotsubo cardiomyopathy: a study of the National Inpatient Sample 2008 to 2009. Am Heart J. 2012;164(2):215-221. https://doi.org/10.1016/j. ahj.2012.04.010.

4. Smilowitz NR, Hausvater A, Reynolds HR. Hospital readmission following takotsubo syndrome. Eur Heart J Qual Care Clin Outcomes. 2018;5(2):114-120. https://doi.org/10.1093/ehjqcco/qcy045.

5. Vallabhajosyula S, Deshmukh AJ, Kashani K, Prasad A, Sakhuja A. Tako-Tsubo cardiomyopathy in severe sepsis: nationwide trends, predictors, and outcomes. $J$ Am Heart Assoc. 2018;7(18):e009160. https://doi.org/10.1161/JAHA.118.009160.

6. Shaikh N, Sardar M, Jacob A, et al. Possible predictive factors for recovery of left ventricular systolic function in takotsubo cardiomyopathy. Intractable Rare Dis Res. 2018;7(2):100-105. https://doi.org/10.5582/irdr.2018.01042.

7. Shah M, Ram P, Lo KBU, et al. Etiologies, predictors, and economic impact of readmission within 1 month among patients with takotsubo cardiomyopathy.
Disclosures: All authors report no conflicts of interest.

Funding: Dr. Pack was supported by the National Heart, Lung, and Blood Institute of the National Institutes of Health, under Award Number 1K23HL135440. Dr. Lagu was supported by the National Heart, Lung, and Blood Institute of the National Institutes of Health, under Award Number K01HL114745. Dr. Lindenauer was supported by the National Heart, Lung, and Blood Institute of the National Institutes of Health under Award Number 1K24HL132008

Clin Cardiol. 2018;41(7):916-923. https://doi.org/10.1002/clc.22974.

8. Misumida N, Ogunbayo GO, Kim SM, Abdel-Latif A, Ziada KM, Sorrell VL. Clinical outcome of takotsubo cardiomyopathy diagnosed with or without coronary angiography. Angiology. 2019;70(1):56-61. https://doi. org/10.1177/0003319718782049

9. Charlson ME, Pompei P, Ales KL, MacKenzie CR. A new method of classifying prognostic comorbidity in longitudinal studies: development and validation. J Chronic Dis. 1987;40(5):373-383. https://doi.org/10.1016/00219681(87)90171-8.

10. Elixhauser A, Steiner C, Harris DR, Coffey RM. Comorbidity measures for use with administrative data. Med Care. 1998;36(1):8-27. https://doi. org/10.1097/00005650-199801000-00004.

10. Templin C, Ghadri JR, Diekmann J, et al. Clinical features and outcomes of takotsubo (stress) cardiomyopathy. N Engl J Med. Sep 3 2015;373(10):929938. https://doi.org/10.1056/NEJMoa1406761.

12. Medina de Chazal H, Del Buono MG, Keyser-Marcus L, et al. Stress cardiomyopathy diagnosis and treatment: JACC state-of-the-art review. J Am Coll Cardiol. 2018;72(16):1955-1971. https://doi.org/10.1016/j.jacc.2018.07.072.

13. Ghadri JR, Wittstein IS, Prasad A, et al. international expert consensus document on takotsubo syndrome (part I): clinical characteristics, diagnostic criteria, and pathophysiology. Eur Heart J. 2018;39(22):2032-2046. https://doi. org/10.1093/eurheartj/ehy076. 